Ebisu Ebisu

Études japonaises Études japonaises

49 | printemps-été 2013

De chose en fait : la question du milieu

\title{
Un essai d'interprétation de la raison des choses selon Yamanouchi Tokuryū : l'appel fait à la philosophie bouddhique
}

An Attempt to Interpret the Reason for Things According to Yamanouchi

Tokuryū and his Use of Buddhist Philosophy

山内得立によるものごとの存在原理の佛教哲学的解釈試論

\section{Frédéric Girard}

\section{(C) OpenEdition}

\section{Journals}

Édition électronique

URL : http://journals.openedition.org/ebisu/781

DOI : 10.4000/ebisu.781

ISSN : 2189-1893

Éditeur :

Institut français de recherche sur le Japon (UMIFRE 19 MAEE-CNRS), Maison franco-japonaise

Édition imprimée

Date de publication : 1 avril 2013

Pagination : $91-113$

ISSN : $1340-3656$

\section{Référence électronique}

Frédéric Girard, « Un essai d'interprétation de la raison des choses selon Yamanouchi Tokuryū : I'appel fait à la philosophie bouddhique », Ebisu [En ligne], 49 I printemps-été 2013, mis en ligne le 19 mai 2014, consulté le 02 mai 2019. URL : http://journals.openedition.org/ebisu/781 ; DOI : 10.4000/ ebisu.781 


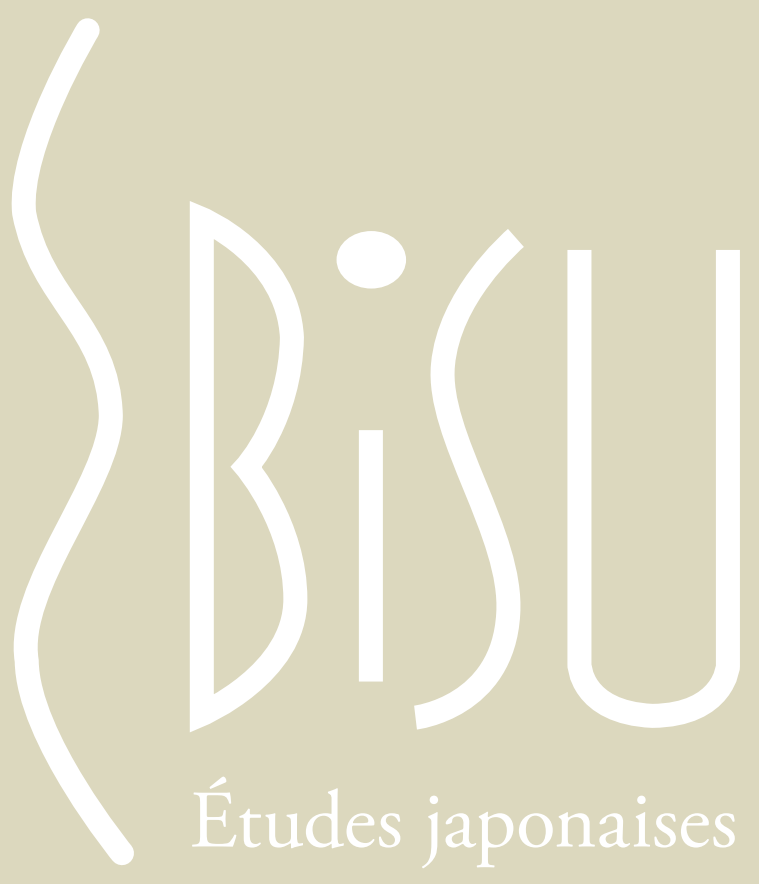

Dossier

De chose en fait : la question du milieu

Articles issus du colloque de Shin-Hirayu

Coordonné par Augustin BERQue

Varia | Nicolas Baumert - Peut-il exister des terroirs du saké ?

Conférences de la MFJ | François Macé - Le Kojiki, une Énéide longtemps oubliée ? | Patrick Beillevaire - Présences françaises à Okinawa : de Forcade (1844-1846) à Haguenauer (1930)

Livres à lire

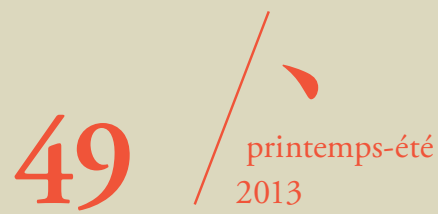




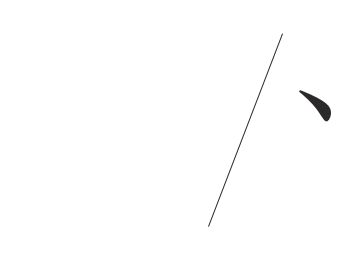

\section{Dossier}

\section{De chose en fait : la question du milieu}

Mono kara koto e to füdo no mondai

物から事へと風土の問題

Articles issus $d u$

colloque de Shin-Hirayu

Dossier coordonné

par Augustin Berque 


\section{Un essai d'interprétation de la raison des choses selon Yamanouchi Tokuryū L'appel fait à la philosophie bouddhique}

Frédéric GIRARD*

\section{Usage philosophique des notions bouddhistes de causalité : l'exemple de Yamanouchi Tokuryū}

I.1. Le présent colloque auquel nous sommes conviés, à l'heureuse initiative d'Augustin Berque, propose une thématique commune autour de Yamanouchi Tokuryū 山内得立 (1890-1982) et de son livre, Logos et Lemme'. Yamanouchi est un penseur conscient de la nécessité d'intégrer les particularismes culturels dans une réflexion à valeur universelle, respectueuse aussi bien de l'élaboration de principes universels admis de tous, que des expressions les plus singulières de la pensée. Il a voulu élaborer, dans une partie de son œuvre qui se réferre également aux sciences sociales, à l'historiographie et à la philologie, une philosophie sui generis, qui se fonde en partie sur la dialectique bouddhique telle qu'elle s'est développée au cours de l'histoire, de l'Inde au Japon, chez les grands penseurs que sont Asangga, Vasubandhu ou Nāgārjuna, ainsi que chez leurs émules chinois. Or, cette dialectique prend tout son sens, non pas dans un ciel des idées ou dans un univers notionnel qui ne renverrait qu'à lui-même, mais dans une expérience vécue qui est celle de la méditation bouddhique. C’est pourquoi

* École française d'Extrême-Orient

1. Rogosu to Renma ロゴスとレンマ (Logos et Lemme), Tokyo, Iwanami shoten 岩波書店, 1974. 
des rapprochements que l'on peut faire avec des concepts européens, tels le principe d'identité ou de contradiction, semblent la plupart du temps glisser sur la réalité des problématiques, car ils présupposent une transparence parfaite du langage et des notions à eux mêmes comme le croyait par exemple un Descartes. Il semble de la meilleure méthode de partir de notions bouddhiques, celles-mêmes que connaissait bien Yamanouchi, pour aborder sa philosophie dans ce qu'elle a de plus original : le dépassement du principe du tiers exclu. C'est pourquoi nous croyons avisé de faire état des doctrines de la causalité selon les perspectives de la philosophie de l'Avatamsaka, qui s'inscrivent dans la lignée naturelle de la philosophie nāgārjunienne, afin d'éclairer le propos de Yamanouchi de la manière la mieux appropriée et pertinente.

Une question préambulaire est de savoir ce qu'est la causalité et la coproduction conditionnée selon la philosophie bouddhique classique, ce qui ne peut être traité ici que de manière succincte. Le sujet est central, les interprétations sont multiples. Proposer sa propre interprétation semble relever de l'arbitraire : on n'aura fait qu'en exposer une de plus, nous dira-t-on ? Cela n'est pas certain, car il semble que la philologie a dans ce domaine fait depuis plusieurs décennies quelques avancées et si les interprétations évoluent c'est plus afin d'écarter des erreurs d'exégèse que pour avancer des hypothèses supplémentaires.

1.2. Dès l'époque de ses premières formulations doctrinales que l'on peut trouver ou reconstituer parmi les textes sacrés faisant état de la " découverte " philosophique du Buddha, c'est-à-dire du contenu même de son Éveil - les Quatre Nobles Vérités et la Co-production conditionnée -, jusqu'aux développements de l'exégèse la plus tardive, il apparaît que les doctrines bouddhiques posent la question de la causalité comme un problème central. Néanmoins, l'on peut se demander si cette causalité recouvre ce que nous avons tendance, dans la philosophie et la science en Occident, à entendre par là. Il importerait de tirer au clair des définitions préalables à toute discussion sur cette notion ainsi que d'autres qui lui sont connexes. Circonscrire en effet des registres de problématiques est primordial avant de poursuivre tout traitement de cette question : registre occidental, registre oriental et en particulier bouddhique. En outre, la pensée bouddhique s'étant développée au cours de plus de deux millénaires dans plusieurs régions géographiques du monde, cette même théorie de la causalité a pris 
des formes variables et a connu des résonances différentes, cela va de soi, mais il est également remarquable qu'elle soit restée persistante et constitue une manière de constante de la pensée et de la doctrine bouddhiques. Une telle pérénité philosophique ne nous assure-t-elle pas de l'existence d'un noyau invariable, ou presque, des conceptions bouddhiques au cours du temps et à travers les régions du globe où elle s'est développée ? N'importet-il pas dans ce cas de définir ce noyau?

\section{Phénoménologie et sotériologie de la causalité dans le bouddhisme}

II.1. Au premier abord, il semble que si la causalité bouddhique cherche à rendre compte du " comment » des choses, elle ne porte pas en elle-même sur leur "pourquoi ». Phénoménologie et sotériologie se côtoient : expliquer les choses est concomitant de la libération de ces mêmes choses en ce qu'elles ont d'asservissant, et l'on peut se demander si l'explication causale même ne revient pas à atteindre la délivrance suprême, ou à se libérer, si ce n'est des choses, tout du moins de ce qui est aliénant en elles. La Stance d'Aśvajit, apprise et récitée par tout religieux au début de sa prise de vœux, déclare en effet :

Des choses (dharma) qui sont nées d'une cause,

Le Tathāgata en a dit la cause,

Ainsi que l'arrêt.

Il a dit ce qu'il en était, lui le grand religieux².

La traduction chinoise de Yijing 義淨 (635-712 ?) rend hetu, cause, indifféremment par cause ou condition, termes qui distingués au départ deviennent synonymes, donnant ainsi le sentiment que la notion de causalité revêt une portée plus générale et universelle encore, portée qu'elle n'avait pas dans l'original :

Toutes les choses sont nées de conditions.

D'elles, le Tathāgata en a exposé les causes,

2. Pratītyasamutpädagāthā, Mahāvagga, I.23.5; Vinaya, I 40 ; Mahāvastu, III 62. 
Ainsi que l'extinction de leurs causes et conditions.

Ainsi l'a expliqué le grand religieux ${ }^{3}$.

Un acte de connaissance est-il synonyme de libération ? La tradition unanime dira qu'il en est la condition nécessaire mais pas suffisante. La religion bouddhique n'est pas une gnose : elle requiert une " mise en œuvre ". La causalité mondaine qui enchaîne l'homme s'ouvre en effet sur une causalité supramondaine où il est en mesure d'évoluer en une souveraine liberté. Le profane est soumis au cours des choses comme à un cercle vicieux, en raison du fait qu'il le subit en fonction de ses affects et émotions; le sage, lui, agit au sein de ce même cours des choses mais en le dominant et en le maîtrisant. Le premier est esclave de ses passions et agit de manière calculée pour obtenir ce qu'il convoite, tandis que le second a la compassion pour motivation de son action si bien que, loin de subir le monde, il agit «sans calcul ni effort » en son sein afin d'y exercer son ouvre bienfaisante.

Il a néanmoins fallu passer d'un registre mondain à un registre supramondain : la mise en œuvre est précisément ce qui assure le passage d'un plan à l'autre. Elle est ascendante dans le premier cas et descendante dans le second. Le rapport entre ces deux types de causalité est ce sur quoi s'est exercée la réflexion bouddhique depuis les premiers temps de son apparition, en en renouvelant la problématique en fonction des milieux où elle s'est déployée : la diversité apparente des formulations de ces doctrines est née de ce fait. On peut situer dans la Chine des $\mathrm{IV}^{\mathrm{e}}-\mathrm{VI}^{\mathrm{e}}$ siècles l'acmé de cette réflexion et d'une transformation, voire d'une révolution métaphysique. L'univers du Nirvāna n'est plus seulement un monde idéal que l'on viserait, il est ce par quoi procède le monde empirique, et c'est la raison pour laquelle le Nirvāṇa n'est pas à chercher ailleurs que dans le Saṃsāra, ni celui-ci dissocié de celui-là.

Cette vision des choses laisse une question métaphysique en suspens : comment passe-t-on d'un plan à l'autre, dans les deux sens ? Tant que l'on

3. 諸法從縁起 如来説是因 彼法因縁盡 是大沙門説. Cette stance est citée comme illustrant la loi de la coproduction conditionnée, yuanqi fashou 縁起法頌, dans la Transmission de la Loi par les Mers du Sud, Nanhai jigui neifazhuan 南海寄歸内法傳, de Yijing, T. LIV, $n^{\circ} 2125$, p. 226c. Elle est, avec les ossements, l'une des deux reliques laissées par le Buddha. Voir Étienne Lamotte, Traité de la Grande Vertu de Sagesse de Nāgārjuna, Bibliothèque du Muséon, vol. 18, Institut orientaliste, Louvain, 1949, réimpression 1967, t. II, p. 631 et p. 1076. 
est dans le Saṃsāra, c'est bien une causalité mondaine, donc du conditionné, qui produit de l'inconditionné, et c'est bien de l'Absolu qui cause du relatif qui est conditionné. Bien souvent, les penseurs sont à cours de langage et de concepts et font appel à des expressions métaphoriques : ils parlent de " saut de côté » (ōchō 横超) dans les écoles de la Terre pure, Jōdokyō 浄土教, d'« Éveil subit » (tongo 頓悟) dans le Zen, de "Révolution du support psycho-somatique " dans les écoles du Rien-qu’information, Yuishiki 唯識, de "fusion parfaite» ( $y \bar{u} z \bar{u}$ 融通, en.yū 圓融) et de « réduction du Triple Monde au mental seul » (sangai yuishin 三界唯心) dans le Kegon 華厳宗, d'unité du mental dans le trichilioégachiocosme (ichinen sanzen一念三千) dans le Tendai 天台宗, de Yoga (yuga 瑜伽) dans le Shingon 真言宗. La doctrine $\mathrm{du}$ " mental un " qui communique aussi bien avec le Buddha qu'avec les êtres sensibles (isshin 一心) est un dénominateur commun à presque toutes les écoles. La préoccupation est en toute occurrence de savoir comment relier ce qui ne peut l'être, le mondain et le supramondain. Une expression résume bien ce souci : inconcevable est la co-production conditionnée, engi nanshi 縁起難思. La causalité du cycle des passions côtoie ou redouble celle de l'Absolu qui répond au phénoménal (shinnyo zuien 真如隨縁).

II.2. Quant à la question du "pourquoi » des choses, autrement dit "pourquoi il est quelque chose plutôt que rien ? ", la tradition considère qu'il importe de la mettre entre parenthèses puisqu'elle n'intéresse pas directement le processus salutaire et n'affecte pas les modalités du " comment " des choses, envisagées en particulier dans sa transition du mondain au supramondain. Ce n'est pas que cette question ait laissé indifférent ou que l'on y ait été insensible : les injonctions à ne pas spéculer sur elles révèlent la pente naturelle de l'être humain à se la poser, parmi les courants d'inspiration bouddhique aussi. On spécule sur la question de savoir si le Buddha lui-même ne tenait pas la " clé de l'univers et de son origine " dans un recoin de son esprit, et que c'est à dessein qu'il n'a pas voulu la révéler à ses auditeurs et à ses disciples afin de ne pas faire dévier les préoccupations de leur esprit d'un champ à un autre. En outre, des théories sur l'origine de l'être sont apparues au sein des courants bouddhiques, même si l'on a voulu, ou cherché, à les juguler et à les critiquer. En outre encore, sur un plan non pas métaphysique mais phénoménologique, l'émergence de l'être est décrite en des termes et suivant des expressions singulières dans un certain nombre de textes qui font songer à la question du "pourquoi " ou à 
une sorte d'étonnement métaphysique devant ce "pourquoi » des choses. La question du " pourquoi » est comme on le voit très subtile : elle tend à être marginalisée, voire obturée, et, lorsqu'elle apparait, la tendance est à ne pas l'aborder de plein fouet.

\section{Réflexions autour des principaux termes bouddhistes de causalité}

III.1. La problématique du passage des " choses " (mono) aux " faits» (koto) est une autre question. Pour le japonisant, il s'agit en premier lieu d'analyser des sémantèmes japonais, des yamato-kotoba, ceux de mono et de koto le cas échéant transcrits par les caractères chinois 物 et 事, et d'examiner leurs relations éventuelles. Il n'est pas assuré qu'on arrive à des résultats pertinents puisque chacun de ces sémantèmes revêt des significations variées qui, en outre, peuvent se recouper. Dans un contexte bouddhique, ces caractères sont lus motsu 物 et $j i$ 事, dans des textes chinois ou sinojaponais, tandis qu'ils peuvent être également rendus mono et koto dans ce même contexte dans des textes japonais ou même sino-japonais. Ils désignent des entités différenciées - motsu des choses ou des êtres, notamment les êtres sensibles; $j i$ le phénoménal en regard du principiel $r i$ 理 sur les relations desquelles, parmi les courants bouddhiques, l'on n'a guère appliqué la réflexion et l'exégèse. Par ailleurs, le terme de ji peut recouvrir celui de motsu assez fréquemment : les choses et les êtres sensibles sont du "phénoménal » par excellence ${ }^{4}$. Nous voyons, en fait, assez mal comment une problématique d'un passage des choses aux faits serait pertinente.

4. Voir dans notre Vocabulaire du bouddhisme japonais, Paris, Droz, 2008, p. 526, la remarque de Suzuki Daisetsu qui implique que $j i$ 事, $u$ 有 et motsu物 sont synonymes chez les traducteurs bouddhiques : "Vastu, fact, event, reality " (Studies in Lan்kāvatārasūtra, pp. 119, 274, $305 \mathrm{f}, 306$ ). "Vastu is a flexible term, generally having $j i$ 事 for it, but its variations are frequently met with. For example, for : vidyate tathatāvastu (Laìkā 147, 6) 有眞如妙物 (Śikṣānanda), 如眞本有 (Bodhiruci), 有事悉如如 (Gunabhadra) ; citravastutva (Laìkāa 108, 8), 種々事物 (Śikșānanda), 種々法體 (Bodhiruci) ; na vastu nāvastu $(\operatorname{Laka\overline {1}} 108,9)$ 非即是物, 亦非非物 (Śikșānanda). Broadly speaking, however, vastu, ji 事, means particular object discriminated by the mind, but it may also designate ultimate reality conceived as object or transcendental intuition. " 
III.2. On remarquera néanmoins que, dans la plus pure veine du taoïsme philosophique dont le Zen sino-japonais s'inspire, une critique virulente est faite de la " chosification" des " choses ", c'est-à-dire des êtres : ceux-ci ne peuvent ni ne doivent être «substantialisés " si l'on veut préserver leur intégrité. C'est non seulement le Zen, mais toute la pensée bouddhique qui d'ailleurs s'insurge contre une telle " chosification " ou "substantialisation ${ }^{5}$ ", sans pourtant porter atteinte à un principe d'identité : une chose ou un être reste lui-même au cours du temps et de l'évolution de son être tout en changeant. La théorie de la vacuité désubstantialise ce que l'esprit humain tend à substantialiser mais, en procédant par un double mouvement de " désubstantialisation » et de "résistance ou d'opposition à une désubstantialisation ", elle maintient un continuum sur l'ossature mouvante de laquelle les êtres se développent, déploient leur action, agissent dans le monde, entrent en relations mutuelles et agissent de manière créative. C'est sous cet angle de vue que l'on peut soutenir que les « choses » deviennent des « faits », des « événements ». Il n'est pas sûr, à nouveau, que la pensée bouddhique ait porté un regard attentif sur ce passage des choses aux faits, qui intéresse à vrai dire deux notions de la philosophie chinoise et en conséquence du bouddhisme fortement sinisé. Il est encore moins sûr qu'une quelconque réflexion, bouddhique ou indigène, se soit appliquée sur le passage des choses (mono) aux faits (koto). Il semble difficile de puiser dans les textes anciens ou plus modernes de l'époque des Tokugawa pour aborder la question. Il s'agit plutôt d'une problématique de penseurs et de philosophes modernes qui tâchent d'aborder des questions de philosophie générale en faisant appel à des termes qui ont une longue histoire au Japon et en Orient, de manière à poser des problématiques à l'aide de concepts indigènes en regard de la tradition philosophique occidentale qui

5. Idem, p. 919, citation extraite du Zhuangzi dans le Traité de Sengzhao, T. XLV, $\mathrm{n}^{\circ}$ 1858, p. 154b3 : "En vérité, la pensée du saint ne chosifie pas les choses ; c’est pourquoi il n'est pas d'illusion qui les saisiraient. "誠以聖心不物於物。故無惑取也。Aussi, p. 56b8-12: "Toutes choses diffèrent mais leur nature est originellement toujours une. Sans pouvoir être des choses, elles n'en sont pas moins des choses qui peuvent chosifier les choses. Aussi bien, le saint ne chosifie-t-il pas les choses sans les chosifier. En ne chosifiant pas les choses, les choses n'existent pas. En n'étant pas sans chosifier les choses, les choses ne sont pas inexistantes. "何者萬物雖殊。然性本常一。不可而物。然非不物。可物於 物。則名相異陳不物於物。則物而即眞。是以聖人不物於物。不非物於物。不物於物。物非有也。 不非物於物。物非無也。 
véhicule, en son fonds, des vocables d'origine grecque pour penser et analyser en quoi consiste le monde. Dans ce sens, il ne s'agit pas d'attacher une importance démesurée au halo sémantique complet que revêtent ces termes, mais de poser la question d'une conception du monde et des choses où ils prennent place de façon cohérente et pertinente, en même temps qu'elle peut être enrichissante pour la réflexion philosophique générale.

III.3. C'est dans ce sens que, pour alimenter la réflexion et les discussions, il serait utile de faire le point sur les principaux termes désignant la causalité dans le bouddhisme sino-japonais : les six causes (rokuin 六因) ${ }^{6}$, les quatre conditions (shien 四縁) ${ }^{7}$, le sens de l'enchaînement des termes de

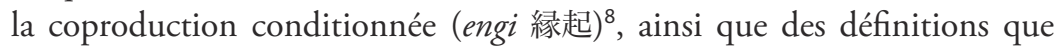
l'exégèse sino-japonaise propose de ces termes. Il ne semble pas inutile non plus de voir comment les notions de puissance immanente et d'actualisation réelle ${ }^{9}$ viennent se greffer, dans l'exégèse sino-japonaise, sur ces notions exprimant la causalité pour donner naissance à de nouvelles conceptions de la causalité : celle d'une interpénétration et d'une interfusion spatiotemporelle universelle qui, elle, rappelle de près le passage de substances à des faits où chacun des deux états est préservé tout en agissant sur l'autre. On peut se demander si ce n'est pas en puisant à des sources d'inspiration telles que l'exégèse bouddhique que des penseurs comme Yamanouchi ont problématisé en termes modernes des schémas de pensée anciens, devenus quelque peu abscons en raison du vocabulaire très spécifique de l'exégèse bouddhique et formulés pour cette raison dans le vocabulaire plus accessible de la philosophie moderne.

6. Idem, pp. 490-492: cause efficiente, nōsain 能作因; cause concomitante, guuin 俱有因; cause analogique, dōruiin 同類因; cause adéquate, sōōin 相応因; cause universelle, hengyōin 遍行因; cause rétributive, ijukuin 異熟因.

7. Idem, p. 198 : condition causale, innen 因縁 ; condition antécédente immédiate, tömugen.en 等無間縁; condition en tant qu'objet, shoen.en 所縁縁 ; condition rectrice, zojjōen 増上縁.

8. Idem, pp. 202-205.

9. On en a un exemple dans la théorie Zen des " cinq stades ", goi 五位 (idem, pp. 315320), qui met en œuvre les concepts liés d'être en soi, tai 体, et de phénomènes pluriels, $j i$ 事. L'examen des quatre champs du connaissable, shikyo 四境, ou les quatre éclairages, shishōyü 四照用, dans le Zen s'y apparente (idem, pp. 1287, 1361). 


\section{La théorie de la coproduction conditionnée et la question de l'origine}

IV.1. Parmi les nombreux métaphysiciens qui se sont posés la question de la raison des choses, on pense presque immédiatement à Leibniz qui a énoncé un principe de raison suffisante (nibil est sine ratione) par lequel il pose qu'un motif, qu'une cause doit être à l'origine de quelque chose qui survient, qui existe, quelle qu'elle soit ${ }^{10}$. Rien n'existe sans cause qui en ait déterminé l'advenue à l'existence (quoddité) ainsi que son mode d'être (eccéité [hoccitas] $)^{11}$. L'existence même d'un être ne peut s'expliquer par le seul enchaînement causal de séries reproduisant les individus similaires ou quasiment identiques d'une même espèce : l'œuf n'explique pas la poule, comme terme originel, mais seulement comme cause seconde ; l'existence de la série prise dans son ensemble, qu'elle soit finie ou infinie, se rapporte nécessairement à une origine radicale. Leibniz l'identifie comme étant ce à quoi la théologie chrétienne attribue le nom de Dieu. Il est l'exister même dont tout être tire son existence par un don gratuit. Il n'est de ce fait qu'une seule raison des choses, dans cette perspective. Leibniz se fait l'interprète d'une tradition philosophique et théologique, qui trouve des échos chez Saint Augustin, Saint Thomas d'Aquin, le Concile de Trente, Descartes. Le principe touche également au mode d'être : il est nécessaire de poser

10. Confession Naturae contra Atheistas (1668), Gottfried Wilhelm Leibniz, Philosophische Schriften, Weidmann, Berlin 1880, vol. 4, p. 105-110. Confessio Philosophi, La profession de foi du philosophe, Texte, traduction et notes par Yvon Belaval, Paris, Librairie philosophique J.Vrin, 1970, p. 47.

11. De la production originelle des choses prises à sa racine, G. W. Leibniz, Opuscules philosophiques choisis, Bibliothèque des textes philosophiques, Librairie philosophique J. Vrin, 1978, en particulier pp. 83-86. Voir aussi le Principe de la nature et de la grâce fondée en raison, Gottfried Wilhelm Leibinz, Euvres philosophiques de Leibniz, avec une introduction et des notes par Paul Janet, Paris, 1900, t. 1, p. 727, \$7 et 8, et La Monadologie : "Nos raisonnements sont fondés sur deux grands principes, celui de la contradiction, en vertu duquel nous jugeons faux ce qui en enveloppe, et vrai ce qui est opposé ou contradictoire au faux, et celui de la raison suffisante, en vertu duquel nous considérons qu'aucun fait ne saurait se trouver vrai ou existant, aucune énonciation véritable, sans qu'il y ait une raison suffisante pourquoi il en soit ainsi et non pas autrement, quoique ces raisons, le plus souvent, ne puissent point nous être connues. " ( $\mathrm{La}$ Monadologie, $\$ 31$ et 32, édition Émile Boutroux, Paris, Delagrave, 1970, pp. 157-158). 
une cause ou un ensemble de causes à la façon dont un individu existe. Les modifications qu'il subit, en particulier, ne sont jamais gratuitement infléchies mais induites en vertu de nécessités, qui se caractérisent par une certaine diversité, mais obéissent à la loi du moindre effort et d'un équilibrage permanent avec le milieu.

IV.2. On sera tenté de dire, au premier abord, que la question de l'origine radicale des choses et de l'être est éludée par la philosophie bouddhique. Elle touche de près à la notion même de dharma, qui recouvre tout un éventail sémantique qui va des choses, des phénomènes jusqu'à leur loi, à l'ordre même de ces choses. Elle recouvre, pour notre propos, deux domaines distincts : le conditionné et l'inconditionné. Le premier est sous l'emprise de cette règle qui veut que tout ce qui est " fait ", " confectionné ", est amené à se défaire ; rien ne dure de ce qui a eu un commencement. Le second échappe au règne du temps mesuré et à sa loi inéluctable qui exige que disparaisse ce qui est né : il est le domaine de l'indestructible, du pérenne, de ce qui n'a pas de commencement parce qu'il n'a pas de fin et inversement. En réalité, de par ces définitions, le second ordre de réalité est-il qualifié ainsi parce qu'il plonge d'emblée dans l'éternité ou bien est-il seulement indéfini dans le temps ? Ce second ordre est-il visé ou est-il acquis d'emblée ? S'il est visé, il restera par définition toujours distinct et séparé de l'ordre conditionné des choses et le sage sera amené à fuir le monde ; s'il est acquis depuis toujours, le même sage pourra vivre dans l'inconditionné tout en vaquant aux affaires de ce monde : il est patent que la seconde option est celle de l'ensemble des courants bouddhiques qui ont prévalu en Extrême-Orient, tandis que le premier y a été considéré comme une sorte d'exception limitée au Véhicule qualifié de Petit ou d'Inférieur.

IV.3. Entre " but ", visée téléologique et point de départ absolu, le choix s'est porté sur la seconde option : en l'occurrence, le terme de " point d'appui » (eji 依恃, eshi 依止) a été largement utilisé sans que l'on dise exactement en quoi il consiste. Ce « point d'appui » n'est pas destiné à rendre compte des choses et de leur état. Il est posé dans son absoluité afin de fournir un repère, un point d'ancrage dans le mouvant des choses, pour éviter de donner à cet état perpétuellement changeant des choses une finalité qui le dépasserait et en quête de laquelle l'être humain serait comme destiné à être dévoré. Ce point d'appui ne peut tenir lieu de point d'origine à partir duquel les 
choses conditionnées seraient comme émanées et où elles seraient appelées à retourner : il est posé comme en «synchronie » (dōji 同時) avec le phénoménal. Ceux qui se risquent à le mettre en diachronie avec le monde conditionné sont immédiatement déclarés "non-orthodoxes " ou " hérétiques ": ils posent un absolu d'où s'écoulerait comme de sa source un monde phénoménal qui serait appelé à s'y résorber à nouveau ; à un temps jadis où régnait une sorte d'Eden métaphysique succède un temps présent fait d'impermanence et de son corollaire, la douleur ou le mal-être. De ce fait, l'absolu sera mis au même plan métaphysique que le phénoménal et perdra son caractère propre qui était précisément d'être " inconditionné ». Certaines doctrines de la Terre Pure n’ont pas échappé à ce vice métaphysique. La pensée bouddhique chinoise a mis en forme philosophique cette question dans la fameuse "Stance sur l'authentique et le fallacieux " (Shinmoju 眞妄頌), composée en Chine et abondamment commentée au Japon.

C'est sous la forme dont Fuli 復礼 (ayant été actif ou ayant vécu sous l'empereur Zhongzong [684-709]) a résumé les six apories qu'elle est la mieux connue (en chinois Zhenwangsong, en japonais Shinmōju) :

La vraie nature des choses est originellement pure

En raison de quoi les pensées erronées naîtraient-elles?

S’il advenait que le faux naisse du vrai

Comment ce faux aurait-il un terme?

S'il est sans commencement, il n'aura pas de fin

Et s'il a une fin il doit avoir un commencement.

Aussi longtemps qu'on y pense, on retombera sur ce raisonnement

Mon vœu est que dans ce but on s'ouvre au sublime mystérieux

Et qu'en l'éclairant, on sorte du cycle des naissances et des morts ${ }^{12}$.

L'absolu y est accolé au relatif comme dos à dos, les deux ne formant que l'envers et l'endroit d'une même réalité dont on ne peut dire lequel précède l'autre : une conversion du regard permet seule d'appréhender l'authentique dans sa plénitude et de se départir du fallacieux qui empêchait de le voir distinctement.

12. Voir Girard, Traité sur l'acte de foi dans le Grand Véhicule, Keiō University Press, 2004 , pp. 196-197. Voir aussi Vocabulaire du bouddhisme japonais, op. cit., pp. 1325-1326. 
IV.4. La question de l'origine des choses n'est pas la préoccupation première de la philosophie bouddhique. C'est celle de la sotériologie qui prime : l'état de coïncidence avec cette pointe de l'absolu qu'est ce point d'appui ne prend de sens que s'il s'accompagne d'une activité dite altruiste de compassion. Le monde est un espace d'expression et d'action de l'absolu : on ne dit pas le pourquoi du monde. Était-il nécessaire qu'il soit pour que cet absolu apparaisse dans toute son intégrité et sa toute puissance ? Nous ne disposons pas de vraie réponse à cette question que la philosophie grecque, notamment celle de Plotin, nous a habitué à penser. Les seuls éléments dont nous disposons sont des remarques incidentes dans les textes : les domaines de Buddha - c'est-à-dire les mondes - sont l'expression de leurs mérites antérieurs à l'état rétributif; ils sont en somme comme la glorification de leurs actes passés qui, en constituant un champ d'action actuel, donne à ce passé une consistance dépassant toutes les dimensions du temps.

Nous n'avons aucune raison de croire que les penseurs qui s'inscrivent dans les préoccupations de la philosophie bouddhique n'aient pas euxmêmes été préoccupés par cette question des origines de l'état des choses phénoménales, mais soit ils n'ont pas abordé la question à l'aide de notions identifiables, soit ils ont volontairement gardé le silence sur la question c'est peut-être la véritable position bouddhique -, soit les textes qui en ont parlé n’ont pas été conservés.

IV.5. Si une origine est proposée au monde conditionné, ne peut-on tout du moins en voir une esquisse dans la doctrine de l'« ignorance " (en sanskrit avidyā, en japonais mumyō 無明) ? C'est bien ce principe qui explique pourquoi l'homme se meut dans l'erreur, la méprise, l'illusoire, qui sont créés par une vue erronée des choses. Mais ce monde de l'illusion est celui du phénoménal en tant qu’il est considéré comme enchaînant et asservissant l'être humain. Cette ignorance peut-être assimilée au premier et primordial des trois poisons (égarement, en sanskrit moha, en japonais guchi 思痴; désir convoiteux, en sanskrit rāga, en japonais don.yoku 頜欲 ; et aversion, en sanskrit dveșa, en japonais shin. $i$ 嘼急) : elle est un principe purement négatif et explique le pourquoi de l'erreur du monde, non pas le monde lui-même dans son principe d'existence à propos duquel rien n'est dit. Mais pour autant que cette même ignorance est mise au principe de la causalité dans son enchaînement (en sanskrit pratìtyasamutpāda, en japonais engi 縁起), il est possible qu'on lui assigne une autre fonction, 
assez souvent méconnue : pourquoi les choses sont-elles ainsi (en sanskrit tathāta, en japonais shinnyo 眞如) ? Rien ni quiconque ne le sait (en sanskrit avidyā, en japonais mumyō). L'ignorance n'est pas définie comme étant une déficience dont l'homme serait responsable, mais comme congénitale à son être et dépassant le champ de son expérience et, donc, de ce qui lui est connaissable. On peut expliquer autant que l'on peut et l'on veut l'état des choses (tathāta), jamais on ne pourra en dire le pourquoi, du moins dans la condition actuelle de l'homme ; peut-être les choses apparaissentelles autrement dans l'état du Nirvāna mais personne n'en a jamais rien dit : cette ignorance-là n'est pas un manque, elle est une " inscience ", une " inconnaissance », le principe en négatif du savoir et de la connaissance, envisagé comme son pôle opposé.

Si c'est une position de base que l'enseignement bouddhique a observée dès le début de taire volontairement la question de l'origine des choses et, partant, de leur raison, il s'est employé à en décrire le comment de manière détaillée mais pas trop non plus : son descriptif ne dépasse pas celui que la nécessité lui dicte de mener l'homme dans l'état de délivrance, qui est son propre but.

\section{Conclusion : de la sotériologie à la métaphysique?}

V.1. Notre meilleur angle d'approche pour résoudre la question de la raison des choses est d'aborder la théorie de la coproduction conditionnée. La chaîne de la coproduction conditionnée en douze membres (jünishi engi 十二枝縁起) est rapportée aux origines mêmes du bouddhisme et est considérée comme constituant la teneur même de l'Éveil du Buddha tout comme les Quatre Nobles Vérités ${ }^{13}$. Les variations de la liste des membres qui la composent dénotent que le lien qui les relie n'entre pas dans un

13. Vérités du mal-être, de son origine, de son extinction et du chemin qui mène à cette extinction. Elles sont qualifiées de nobles par extension : les deux premières sont mondaines donc profanes, seule la troisième est supramondaine donc noble, et la quatrième, qui mène du mondain au supramondain, participe des deux. C'est en réalité la quatrième seule qui est caractéristique de la religion bouddhique. Alfred Foucher, La Vie du Bouddha d'après les textes et les monuments de l'Inde, Payot, 1949, pp. 201-203. Voir aussi Vocabulaire du bouddhisme japonais, op. cit., pp. 1359-1361. 
cadre déterministe, mais souligne les conditions sine qua non d'apparition des membres subséquents. Elles expliquent le fonctionnement des choses en tant que l'homme a à se libérer de ses passions en vue de l'obtention du Nirvāna et met en évidence le fait que les choses comme les êtres constituent des continuums plutôt que des entités. Elles sont censées expliquer le pourquoi de l'homme dans son existence empirique et son mode d'être. La visée de cette chaîne est à la fois de rendre compte de l'homme dans son existence présente, mais aussi et surtout de lui fournir les moyens de se libérer de ce qui l'asservit dans celle-ci : elle est d'un ressort à la fois mondain et supramondain, tout comme le sont les Quatre Nobles Vérités ; parmi celles-ci en effet, la douleur et son origine - la soif du désir - appartiennent au mondain, tandis que la cessation de la douleur ainsi que le chemin qui y mène appartiennent au domaine supramondain. L'analyse du réel dégage l'existence d'éléments mondains, les choses conditionnées, celles qui sont soumises à la naissance et en conséquence à la disparition, celles qui sont composées, et celle d'éléments supramondains, les inconditionnés, ceux qui sont du ressort de l'absolu, parmi lesquels le Nirvānạa, la Prajñā, l'Espace, la Talité ${ }^{14}$. L'homme est invité à chercher et à trouver en lui-même ce qui échappe à la naissance et à la mort pour assurer son salut, et toute sa quête est orientée par la découverte de cet élément.

V.2. Le tout de cette chaîne tourne autour de l'élément de complexion, de structure dynamique et organique (en sanskrit samskāra, en japonais gyō 行), qui fait se mouvoir les êtres. De cette complexion, nul ne peut en indiquer l'origine, tout comme personne ne peut remonter à l'infini dans la chaîne de l'œuf et de la poule : on ne sait rien de son pourquoi (en sanskrit avidyā, en japonais mumyō 無明), ainsi que peut se laisser entendre le premier élément de la série en tant qu'aveu d'ignorance, en dehors de son sens le plus couramment admis d'" ignorance ", c'est-à-dire de connaissance erronée

14. Le Nirvāna définit l'extinction suprême, qu'elle comporte encore des reliquats passionnels ou projections ou non ; la Prajn̄ā est la connaissance indistinctive supramondaine portant sur la Vérité, qui est de nature intuitive ; l'Espace est non pas l'espace local mais le "lieu indistinct où les choses n'entrent pas en obstruction mutuelle ; la Talité est la manière d'être des choses qui actualise leur aspect authentique dans le phénoménal. Voir André Bareau, L'absolu en philosophie bouddhique : évolution de la notion d'asamskṛta, Paris, 1951. Thèse non publiée. 
portée par les passions et les affects. Les autres points culminants de cette série sont le désir qui s'attache aux aspects agréables des sensations. Ce désir projette des entités fictives, des semblants d'êtres que les désirs font prendre pour des réalités, mais entités agissantes qui poussent l'homme à faire naitre ces semblants en tant qu'objets stables, ce qu'ils ne sont par définition pas. Or, c'est un axiome de base, ce qui naît en raison d'une composition d'éléments est amené par la force des choses à se défaire, si bien que la mort, douzième élément, en est nécessairement le terme. Là s'arrête la liste des douze termes mais la série se poursuit, dans ses formulations les plus anciennes, par l'énoncé d'états psychologiques pénibles associés à la mort, qui constituent pour les survivants une douleur, sinon même la douleur par excellence : chagrin, tristesse, regret, lamentation, désespoir. Cette série, dans sa formulation indienne, a été interprétée avec un certain succès et beaucoup de conviction, dans un sens freudien par Alfred Foucher ${ }^{15}$. C'est la libido qui l'explique et le choix du vocabulaire indien utilisé va dans le sens de cette interprétation.

V.3. Dans cette série, un terme névralgique est celui de pratyaya en sanskrit, en 縁 en japonais, souvent rendu par condition, mais qui désigne plutôt un point d'ancrage et d'appui. Il indique ce qui permet de relier les membres de la chaîne entre eux. Lorsque la chaîne est envisagée à rebroussepoil, c'est l'aspect relationnel qui est mis en évidence dans la perspective de la délivrance du cercle vicieux des phénomènes qui se produisent et disparaissent, le samsāra, tandis que considérée dans le sens du poil, ce sont les pseudo-entités qui apparaissent en pleine lumière ${ }^{16}$. Nous prenons le terme de samsāra dans l'acception qu'en donne par exemple Foucher de "flux universel du perpétuel devenir ${ }^{17}$ ", de flux mental soumis à des passions asservissantes, plutôt que dans le sens de " transmigration " qui ne semble pas avoir eu beaucoup de prise dans les vocables sino-japonais : pour autant que le mental est agité, l'individu est asservi au monde, qui est saṃsārique, dès qu'il est apaisé, il vaque librement dans ce même monde, qui est nirvāné. Deux directions sont indiquées.

15. Alfred Foucher, La Vie du Bouddha d'après les textes et les monuments de l'Inde, Payot, 1949, pp. 163-166.

16. Sur ces deux orientations, idem, p. 170.

17. Idem, p. 354. 
V.4. Le Mahāyāna (Grand Véhicule) donnera à ces deux orientations la valeur de deux niveaux d'être, si l'on veut bien nous autoriser à utiliser cette expression qui nous semble appropriée en la circonstance : l'adéquation qui est faite entre le Saṃsāra et le Nirvāṇa ne les érige pas en deux éléments distincts mais comme deux plans qui, n'étant que l'envers et l'endroit d'une même réalité, sont posés comme deux niveaux tout en ne constituant qu'une seule réalité. La délivrance est entièrement intériorisée dans cette perspective. Les Buddha, qui incarnent la Loi - ce qu'indique l'expression de corps de la Loi - vaquent à leur activité altruiste en déployant des corps de métamorphose ou de correspondance afin de s'adapter aux besoins des êtres sensibles en proie à la douleur ou au mal-être de leur existence. Un plan de jouissance des joies de l'expérience de la Loi est ménagé, dans lequel les Buddha partagent leur expérience avec d'autres Buddha ou des bodhisattva, dans un corps dit de rétribution ou de fruition pour soi-même ou pour autrui. Dans ces niveaux, les fonctions assumées par les Buddha sapience, compassion, médecin - sont déléguées à des bodhisattva qui les exercent en acte auprès des êtres qu'ils ont à convaincre et à délivrer de leurs erreurs et de leurs douleurs. Ils jouissent de la sphère de la Loi et en font partager les bienfaits en endossant les rôles de Buddha et en prêchant cette Loi à l'aide d'un langage articulé ou d'actions symboliques.

Dans ce nouveau contexte, la coproduction conditionnée explique les choses nées, conditionnées, et se résout dans le domaine du non-né ou de l'inné. Les choses sont évacuées de leur être (être) comme de leur non-être (néant), pour se présenter sous un mode d'être évacué de toute substantialité comme de toute projection ou finalité médiatisée, mode d'être que définit une voie médiane qui se tient à l'écart de toute prise de position affirmative tranchée. Elle réfute toute thèse radicale se prononçant sur l'être ou le non-être des choses, fût-elle négative dans un ni... ni... apophatique, afin de ménager la thèse d'un être partiel ou d'un non-être partiel qui est la seule option permettant que prenne place celle d'un continuum des choses. Le continuum - en réalité un continu discontinu - est à son tour la seule hypothèse qui rende compte de l'être des choses qui sont envisagées en tant que phénomènes engagés dans la temporalité et dont l'homme ne peut rien dire qui dépasse le champ de son expérience sensible. Les quatre propositions (catuṣkoțī, japonais shiku 四句) du théoricien indien du Mādhyamaka, Nāgārjuna (II ${ }^{\mathrm{e}}$ siècle de notre ère ?), - ni être, ni non être, être et non-être à la fois, ni être ni non-être à la fois - en sont une formulation qui prévoit 
tous les cas de figure pensables de cette volonté de désubstantialiser ${ }^{18}$. Une autre approche en est la formulation de huit négations (happu 八不) $)^{19}$, qui est le produit d'une extrapolation sinisée des énoncés de Nāgārjuna et non pas à proprement parler de ce philosophe, bien que la tradition sinojaponaise le donne pour son initiateur. Suzuki Daisetsu a de son côté rendu compte de cette phénoménalité des choses d'un point de vue qui réduit le tétralemme au troisième membre ${ }^{20}$. Une chose est à la fois elle-même et ce qu'elle n'est pas, ou mieux, ce qu'elle est indissociable de ce qu'elle n'est pas, de ce qui n'est pas elle, sokuhi 即非. Ici, le préfixe soku indique un rapport d'indissociabilité entre deux éléments (furi 不離) et non celui d'une union substantielle de deux entités (göitsu 合一) qui en créerait une troisième, bien encombrante du point de vue bouddhique : tel est du moins le point de vue de Nishida Kitarō 西田幾多郎 (1870-1945) à l'encontre de celui de Tanabe Hajime concernant ce préfixe. C'est en effet ce que l'on peut inférer de plusieurs assertions de Nishida disséminées dans son œuvre : loin de désigner une unité (göitsu), soku se dit d'une auto-identité contradictoire $^{21}$; l'Absolu ne s'oppose pas au Relatif dans un rapport de corrélation mutuelle (sōsoku 相即) comme deux entités en vis-à-vis, mais au sein d'une auto-identité des contraires absolus, ce qui suppose que l'Absolu est $\mathrm{Un}^{22}$. Or cette auto-identité des contradictoires absolus, qui est à la fois non-être absolu et être, est une indissociabilité immédiate (suyū furi 須失不離) ${ }^{23}$. On retrouve la définition même du préfixe soku comme indissociabilité, définition exacte car elle s'applique à une seule réalité, en

18. Voir Guy Bugault, article "Catușotī " de l'Encyclopédie de philosophie universelle, vol. II, t. 2, pp. 2820-2821 ; idem, L'inde pense-t-elle?, Paris, PUF, 1994, pp. 242, 246248, etc. Cette façon de penser les catégories en tétralemmes était courante en dehors de Nāgārjuna, L'Abhidharmakośa de Vasubandhu, traduit par Louis de La Vallée Poussin, Mélanges chinois et bouddhiques, vol. XVI, Institut belge des hautes études chinoises, Bruxelles, 1971, II, p. 26 ; III, pp. 116-117.

19. Happu 八不: fushō 不生, non-naissance ; fumetsu 不滅, non-destruction ; fudan 不斷, non-élimination ; fujō 不常, impermanence ; fuitsu 不一, non-identité ; fui 不異, nondiversité ; furai 不來, non-arrivé ; fuko 不去, non-parti.

20. Voir Frédéric Girard, "La Philosophie au Japon », Encyclopédie philosophique universelle, Le Discours philosophique, PUF, 1998, pp. 600 et 614.

21. Shü no seisei hatten no mondai, 1937, NKZ, VIII, pp. 524-525.

22. Lettre $\mathrm{n}^{\circ} 1380$, du 21 septembre 1939, NKZ, XIX, p. 86.

23. Lettre ${ }^{\circ} 1789$, du 8 juillet 1943, NKZ, XIX, p. 246. 
regard de son interprétation déviante comme unité de deux êtres, car elle pose deux entités abstraites ${ }^{24}$. Néanmoins, cette vision des choses, même radicalement insubstantielle, amène à penser qu'un principe du " tiers inclu " serait le meilleur mode d'approche logique de la réalité. C'est ce que Yamanouchi soutient dans son ouvrage pionnier. Certains penseurs bouddhiques ne pourraient-ils souscrire à cette option intellectuelle qui rompt avec le logicisme aristotélicien, depuis la découverte de la matièreénergie grâce à la physique quantique ${ }^{25}$ ? Le logique, qui est censé recouvrir les modes opératoires du mental et du pensable, ne serait-il pas, selon lui, à mettre en corrélation avec le réel que nous livre l'expérience?

L'approche négativiste que nous avons brossée, née en Inde, a été introduite en Chine, où elle a fait l'objet d'une étude dans les milieux monastiques, mais n'a pas véritablement eu les faveurs des adeptes ni des théoriciens, qui leur ont préféré des approches et des formulations positives, comme le sont celles de la théorie de l'embryon de Tathāgata (nyorai 如来 en japonais, traduit parfois par l'ainsité), de la nature de Buddha, du véritable aspect des choses, autrement appelé «Talité ».

V.5. La coproduction conditionnée se laisse analyser, dans ce contexte du Grand Véhicule en deux perspectives ou niveaux :

1/ Une coproduction conditionnée émanant du monde des choses et de leur Loi, fajie yuanqi 法界縁起, en épousant les conditions, zuiyuan yuanqi 隨縁縁起, afin d'épouser les formes, les souhaits, les facultés mentales des êtres.

2/ Une coproduction émanant du monde des choses et de leur Loi, en se laissant séduire par le conditionné. Cette doctrine, pour le moins étrange

24. Voir notre Vocabulaire du bouddhisme japonais, Paris, Droz, 2008, pp. 1476-1480. Nous avons marqué cette position de Nishida dans "Logique du lieu et expérience unitive de l'absolu ", in Augustin Berque, Logique du lieu et dépassement de la modernité, vol. I, Nishida : La mouvance philosophique, Ousia, 2000, pp. 225-226 et 235.

25. Il faut rendre justice à un épistomologue et logicien qui a mis en évidence des principes, fondés sur les sciences, qui pourraient rejoindre les préoccupations et les positions des problèmes tels que la philosophie bouddhique les envisage et les aborde. Stéphane Lupasco considère, en effet, que "Le principe de complémentarité contradictoire doit remplacer le principe de non-contradiction comme fondement du logique ", et que le principe du " tiers inclus ", qui englobe les contradictoires, peut avec profit être intégré dans la logique. 
est celle du "parfumage », junji 薰習, de la Talité par le conditionné, zuiyuan zhenru 隨縁眞如.

À un stade d'élaboration ultérieur, cette analyse de la production des choses donnera naissance à la théorie des quatre sphères de la Loi (sifajie 四法界) - terme qui désigne à la fois le monde des êtres et des choses et celui de la loi qui les régit :

- le plan des choses phénoménales, shifajie 事法界;

- le plan nouménal du principe des choses, lifajie 理法界;

- le plan de l'union du principe et des phénomènes, lishi fajie 理事法界;

- le plan dans lequel les phénomènes ne se font plus obstacles entre eux, shishi wu'ai fajie 事事無碍法界.

Deux niveaux sont analysés en quatre moments dialectiques. D’autres analyses sont proposées par les théoriciens des écoles bouddhiques, mais celleci semble avoir retenu l'attention de philosophes comme Nishida Kitarō, notamment dans son article inachevé « À propos de ma logique ${ }^{26}$ ».

V.6. Ces deux niveaux de réalité sont mis en équation de manières différentes dans l'école de l'Ornementation fleurie, Huayan 華厳宗, qui est un enseignement particulier du Grand Véhicule, basé sur le sûtra du même nom :

1/ La conscience-de-tréfonds (alāyavijñāna) est dite identique à tous les phénomènes qu'elle contient, selon Zhiyan 智偘 (602-668) et Fazang 法蔵 (643-712), qui parlent à ce propos de " profondeur extrême de la coproduction en dépendance " (yuanqi zhenshen 縁起深甚), en reprenant une thèse propre à Tanqian 曇遷 ( $\mathrm{vI}^{\mathrm{e}}$ siècle).

2/ La même expression de "profondeur extrême de la coproduction en dépendance " est utilisée par Usang à propos de la fusion mutuelle, xiangrong 相融, de la Talité et de l'Inscience, dans un sens similaire à la Méthode d'apaisement et d'examen mental selon le Grand Véhicule (Dasheng zhiguan famen 大乘止觀法門), texte que l'on peut attribuer au même Tanqian et non à Huisi 慧思 $(515-577)^{27}$.

26. Nous avons traduit cet article de mai 1945 dans "Logique du lieu et expérience unitive de l'absolu ", op. cit., pp. 243-244.

27. Des travaux récents ont montré que ce texte est de la lignée de l'Ornementation fleurie et non du Tiantai, comme on l'a longtemps cru. Son attribution à Huisi du Tiantai est controuvée. 
V.7. La théorie des trois natures de l'école Yogācāra a été utilisée par le Zhiyan afin de développer sa doctrine de la vacuité et d'exposer sa conception de l'interpénétration universelle.

La nature dépendante (yituoxing 依他性) se fonde sur la nature imaginaire (bianjixing 遍計性) : si les choses apparaissent en dépendance c'est qu'on les imagine telles. Cette nature imaginaire des choses n'étant que le produit d'une imagination fausse, elle n'existe pas. D'où il suit que la nature dépendante n'existe pas non plus. Cette non-existence de ces deux natures définit précisément la nature parfaite, yuanchengxing 圓成性.

Ces trois natures sont comprises intellectuellement (jie 解) ainsi que par la pratique (xing 行), selon Zhiyan. La réalisation des trois natures par la pratique permet d'appréhender la Talité en tant qu'état véritable des phénomènes qui ne sont ni existants, you 有, ni non-existants, $w u$ 無. Zhiyan a remplacé le terme d'existant par celui de phénomène ou de donnée factuelle, shi 事, et celui de non-existant par celui d'absolu ou de principe des choses, $l i$ 理. Zhiyan opère un passage entre la notion d'être ou d'étant et celle de phénomène ou de fait. La Talité devient, dans ce nouvel ensemble sémantique sinisé, un état de non-différentiation entre phénomènes et absolu ou entre faits empiriques et principe des choses. Mais cette nondifférentiation a pour corollaire que le phénoménal est lui-même l'absolu. Il suit de là qu'il n'y a pas d'obstruction entre les phénomènes puisqu'ils recèlent en eux l'absolu. Cet état des choses est qualifié de "non-obstruction des phénomènes entre eux" (shishi wu'ai 事事無碍). Le terme même de "non-obstruction » est celui par lequel se définit précisément l' "espace » bouddhique, non pas celui orienté du domaine conditionné, deśa, fang 方, mais celui absolu de l'inconditionné, ākāśa, xukong 虚空. Sans le dire explicitement, Zhiyan, par cette théorie de la non-obstruction des phénomènes, décrit ni plus ni moins que l'absolu lui-même dans son mode d'être. Usang et Fazang suivent Zhiyan mais n'expliquent guère concrètement en quoi consiste cette non-obstruction, si ce n'est par la théorie de la coproduction conditionnée. Celle-ci se trouve de ce fait portée au plan de l'absolu et l'absence d'explicitation concrète de cette non-obstruction phénoménale permet toutes les interprétations possibles de ce qu'est la coproduction conditionnée : elle perd de plus en plus le caractère premier de libération des passions auxquelles l'homme est en prise pour revêtir celui d'une sorte de loi universelle qui régente les phénomènes, quels qu'ils soient, et leur confère un caractère inconditionné. 
V.8. La coproduction conditionnée du Huayan est envisagée dans le champ de la non-obstruction du phénoménal. En effet, le phénoménal se produit en dépendance de causes et de conditions (pratītyasamutpāda). Il est donc analysé sous les espèces de causes, de conditions et d'effets. On a des des causes et des conditions des listes de six et de quatre, qui se recoupent partiellement. L'on conçoit également que les unes incluent les autres à titre de possibles ou d'actualisation à des degrés divers. La non-obstruction des phénomènes est analysée sous deux aspects :

1/ La non-obstruction entre causes et conditions.

2/ La non-obstruction entre causes et effets.

Ici Zhiyan fait intervenir les concepts chinois de "substance » ou d' « êtreen-soi », $t i$ 體, et de " pouvoir » ou d'" activité », de mise en œuvre, yong 用.

$1 /$ Une condition ne produit jamais d'effet sans cause et une cause ne produit jamais d'effet sans condition. Une cause seule ou une condition seule peut donc produire un effet. Le pouvoir de la cause et celui de la condition ne coexistent jamais : lorsque l'une agit, elle est censée contenir le pouvoir de l'autre. Cette inclusion réciproque du pouvoir de la cause et de la condition définit la "pénétration mutuelle ", xiangru 相入.

2/ Le pouvoir étant inhérent à la substance, la proposition précédente conduit à l'inclusion réciproque de la substance de la cause et de la condition, ce qui qualifie l'«identité mutuelle ", xiangji 相即.

Ces explications philosophiques de Zhiyan pour expliciter la notion de non-obstruction des phénomènes font intervenir les notions de causes et de conditions ainsi que d'effets causaux, dans une perspective générale intéressant la légalité universelle et cosmique. Il a été suivi par Usang 義相 (625-702).

Le phénoménal analysé en causes et conditions est constitué de choses et d'êtres qui s'incluent mutuellement, selon un rapport de l'un au tout. Un phénomène contient tous les autres et réciproquement, si bien que ces rapports d'inclusion sont indéfinis. Ils sont qualifiés d'inépuisables. Fazang parlera à leur propos de coproduction conditionnée inépuisable, wujin yanqi 無尽縁起.

V.9. La cause et l'effet explicitent, eux, le phénoménal en tant qu'il évolue au cours du temps. Concernant l'absolu, il n'y a pas d'écoulement mesuré du temps ni de changement afférent dans l'être ; il n'y a en conséquence pas non plus de cause ou d'effet qui entraîneraient une obstruction mutuelle 
dans l'être : l'homme profane est donc tel quel un Buddha, déclare le subitisme du Zen ainsi que l'enseignement final, du point de vue de l'absolu.

Le Huayan déclare, lui, que l'homme ordinaire est un Buddha, du point de vue du phénomène même puisque celui-ci est l'absolu. En apparence, la causalité est niée au sein du phénoménal, non pas en récusant l'existence d'une cause ou d'un effet, ce qui serait saper les fondements mêmes de l'enseignement bouddhique, mais en disant que seule la cause ou seul l'effet existe : un individu est pour lui à la fois et simultanément un homme ordinaire et un Buddha. Quand l'un existe l'autre n'existe pas, car il est considéré comme étant contenu dans l'autre. Zhiyan qualifie cet état de fait par l'expression de "pénétration mutuelle et d'identité mutuelle de la cause et de l'effet ", yinguo xiangru xiangji 因果相入相即, état qui n'est réalisé que par qui a appréhendé la Talité, c'est-à-dire l'état où l'absolu et le phénoménal ne sont plus différenciés, état qui n’a ni commencement ni fin. Il est acquis au stade de complétion de la foi et de non-régression : l'absolu, la Talité, émerge dans le phénoménal à tout instant, de manière comme incongrue, par un acte sans plus aucune intervention de causalité temporelle ou diachronique. L'expression du Huayan qualifie cette émergence continue et instantanée d' "émergence de la nature ", xingqi 性起. De cette expression a disparu le terme de Tathāgata qui s’appliquait à la nature, nature de Tathāgata, si bien que cette nature peut désigner celle de tout être quel qu'il soit en le portant à l'état d'absolu ${ }^{28}$.

\section{VI.}

Qui réalise la Talité est dit être un Buddha de toute éternité depuis le temps passé infini et à chaque moment présent, temps qui s'écoule dans le phénoménal. Il est en même temps qualifié d'homme ordinaire. Zhiyan récuse de ce point de vue une conception de la causalité phénoménale selon laquelle seule existerait une cause ou un effet simultanément. Il a été suivi en cela

28. Nous nous reportons à certaines des analyses d'Ōtake Susumu 大竹晋 concernant les notions de non-obstruction et celles connexes d'interpénétration spatio-temporelles, dans son ouvrage, Shoki kegon kyoggaku no kenkyü 初期華㛜教学の研究, Daizō shuppan 大蔵出版, 2007. 
par Usang et Fazang dans cette version Huayan de la négation de la causalité opérée par le Mādhyamika (l'école du Milieu de Nāgārjuna). L'analyse de ces concepts qui définissent une philosophie de l'interpénétration de l'un et du tout, qui a peut-être fasciné Nishida, a revêtu un caractère métaphysique que, à l'origine, elle n'avait pas. Leur champ d'application se limitait à la causalité envisagée du point de vue du " possible » et de l' « actuel »: une condition est contenue "potentiellement " dans une cause, un effet est compris " potentiellement " dans une cause. Cette "potentialisation " poussée à l'extrême conduit au monde virtuel de l'interpénétration universelle, sur la base d'une présence actuelle de l'absolu dans le relatif. Cette conception n'a-t-elle pas été interprétée dans une optique métaphysique par les philosophes modernes, qui en ont oublié la portée originellement sotériologique que seul Suzuki Daisetsu avait encore en tête lorsqu'il critiquait les « ratiocinations » de Nishida? 